\title{
USING THE VIDEO ANALYSIS IN AEROBIC GYMNASTICS
}

\author{
Salvatore Napolitano, Daniela Tursi \\ University of Naples "Parthenope" - Department Physical Education and Wellness \\ Corresponding: salvatorenapolitano1@alice.it
}

\begin{abstract}
The Aerobic Gymnastics is a complex sport and the movements are performed continuously, intensely at high speed with the musical accompaniment. One can directly assess the overall performance to the naked eye, but is not able to assess the individual elements of movement and technical aspects. The video analysis indirectly, through the ability to stop and review the various stages of movement several times, facilitates the evaluation. The aim of this study is to verify whether the use of video analysis in daily training activities can facilitate the evaluations of the coaches. 4 female athletes will be evaluated using the tabs in the Code of Points with annotations for deductions (0.10 slight error, mean error 0:20, 0:50 fault) and after 30 sessions in two different ways.
\end{abstract}

The athletes are divided into two groups:

1) Control which continues to be evaluated with the traditional forms,

2) Experimental which is evaluated through the use of two cameras, which apply the points of repelle on specific anatomical points.

At the end all the athletes will be traditionally evaluated and one will compare the assessments to highlight the difference between the two groups. The athletes in the experimental group improved at 0.30 and 0.40 compared to initial assessments made without the video analysis and compared to the control group. The experimental group compared with the control group has a better final evaluations in the matter of execution and cleanliness of the gesture. Probably the rapidity of correction of the act requested by the coach after watching the video and the subsequent execution of the athlete support proper execution. This new training methodology may be also tested on athletes, in order to allow a selfassessment through the measurement of the movie and the subsequent correction of performance so that it can better understand the errors committed and implicitly suggest the correction. The simultaneous use of video analysis by athletes and coaches during the training could further improve the result.

\section{Indexing terms/Keywords}

Motor Imagery; Video Analysis; Didactics

\section{Academic Discipline And Sub-Disciplines}

Performance analysis sport education

\section{SUBJECT CLASSIFICATION}

Physical Education

\section{TYPE (METHOD/APPROACH)}

Experimental; Etude case

\section{Council for Innovative Research}

Peer Review Research Publishing System

\section{Journal: Journal of Social Sciences Research}

Vol. 5, No. 2

Jssreditor.cir@gmail.com

www.jssronline.com 


\section{INTRODUCTION}

In Aerobic Gymnastics the introduction of video analysis is of fundamental importance because the elements of difficulty are performed quickly and the coach during the training - in order to analyze them in detail - needs support of the video to slow down, analyze it step by step and identify the mistakes made during the performance. The Aerobic Gymnastics is the ability to perform continuous complex movement patterns originate from traditional aerobic exercises, high intensity, in perfect integration with the accompaniment of music (Code of Scores, 2012). Given the complexity and the speed with which one performs the technical elements of Aerobic Gymnastics, the introduction of video analysis is essential for a qualitative and quantitative evaluation of athletes' performance during training. "The performance analysis can allow the analysis and careful explanation of the evolution of a dynamic and historical motor and sports phenomenon " (Hughes and Bartlett, 2002). "The notational analysis is used by technicians to have an objective analysis of the performance. Tactics, technique and individual movements can be analyzed helping technicians and athletes to re-evaluate their performance and gain advantage during competition "(Hughes and Franks, 2004). Finally, with the motor imagery the analyzed athletes will imagine themselves performing the action of the motor gesture just displayed with the video analysis trying to correct the error in mind, schedule and execute the item properly. Experimental studies have demonstrated the validity of motor imagery in motor learning, defined as a dynamic study during which the subject mentally simulates an action, there is a reactivation of kinesthetic memory allowing you to "relive" the experience of movement. It is a complex cognitive process that encloses the various steps: motor observation, the motor image and motor execution. The motor imagery allows to imagine a movement of a body part without being actually move. There are two modes: the first-person perspective, in which the subject imagines himself performing a movement trying to feel the kinesthetic and motor sensations, and the third-person perspective in which the subject imagines another person performing the movement focusing the attention to the visual-motor aspects (Raiola et al. 2012). According to Sanders, the motor imagery, in conjunction with a limited practice, leads to the same levels of performance obtained with the continuous physical practice (Raiola 2012). The aim is to explore the new way for monitoring and self-evaluation, step by step, throughout an alternative approach that involves the athletes and trainer in a same process on two different point of view. In the same time, it could be empirically investigated the motor skills in different approach on motor control and learning (Raiola 2013) following the specific aspects of each sports discipline.

\section{METHODS}

Given the abundance of the elements of difficulties in Aerobic Gymnastics Code of Points, we will focus a required element for the category junior A (athletes aged 12 to 14 years). The athletes that we will consider are 4 and each of them practices this sport for about two years. We will divide the athletes into two groups: the experimental group, consisting of 2 athletes, will be evaluated with the video analysis and the practice of motor imagery, and the control group will continue its evaluation always with the video analysis and through the compilations of forms by the coach but without reviewing the video and mistakes. The experimental group once analyzed the video of their performance and after identifying the error (motor observation), with the help of the coach will start to imagine himself (first-person perspective of the image motor) performing the gesture and centering the attention to the error committed on their own and trying to correct kinesthetic mentally. Once imagined, the athlete can perform the movement by making changes in the way they have been developed in mind (motor execution). The control group will perform the same element but with the difference that it will be analyzed only by the coach without special references to the athlete on the mistakes made. The element taken into consideration is $1 / 21 / 2$ tuck jump, an element belonging to the group of aerobic jumps in which the athlete must perform a $360^{\circ}$ turn and during the flight must raise both knees to the chest in the collection position. The evaluation will be 20 in 2 hours of training 3 times a week.

\section{RESULTS}

The results highlight errors commonly present during the execution of this element. All athletes at the beginning of the experimental project showed common errors, that is, the failure to achieve the knees to the chest, not the completeness of the $360^{\circ}$ turn at the beginning and at the end of the jump, misalignment of the arms of the shoulders in the preparation phase the jump. After analyzing both groups, we see that the experimental group has improved much faster compared to the control group, because the experimental athletes have found improvements already in its fifth assessment, while for the same improvements the control group will have to wait for the tenth assessment. The tables below highlight a series of improvements to both attest the validity of the video analysis during aerobic workouts and demonstrate how a good practice of motor imagery may increase even more these results. The experimental group has in fact increased his score with the introduction of observation imagery on first-person. While the control group improved by a few tenths compared to the beginning, the experimental group has doubled and in some cases tripled their score.

In the first tables are reported the results that the coach scored with video analysis in the case of an athlete in the experimental group and the results obtained from an athlete of the control group without video analysis comparing then with a histogram of the improvements obtained from both groups. Instead in the other tables are reported the results obtained by the two groups evaluated both with the video analysis with the introduction of motor imagery in the first group. 
Table 1 - First and last assessment with video analysis

First evaluation with video analysis

Name of difficulty Value of difficult

1/2 tuck jump 1/2

0,4

Score from 0 to 10 Deduction

TOT.

Twentieth evaluation with video analysis

Name of difficulty Value of difficulty

Score from 0 to 10 Deduction

TOT.

$1 / 2$ tuck jump $1 / 2$

0,4

8

0,1

0,3

Table 2 - First and last assessment with video analysis.

First evaluation without video analysis

Name of difficulty Value of difficulty

Score from 0 to 10 Deduction

TOT.

1/2 tuck jump 1/2

0,4

5

0,3

0,1

Twentieth evaluation without video analysis

Name of difficulty Value of difficulty Score from 0 to 10 Deduction

$1 / 2$ tuck jump $1 / 2$

0,4

6

TOT.

$0,2 \quad \mathbf{0 , 2}$

\section{Graph n.1}

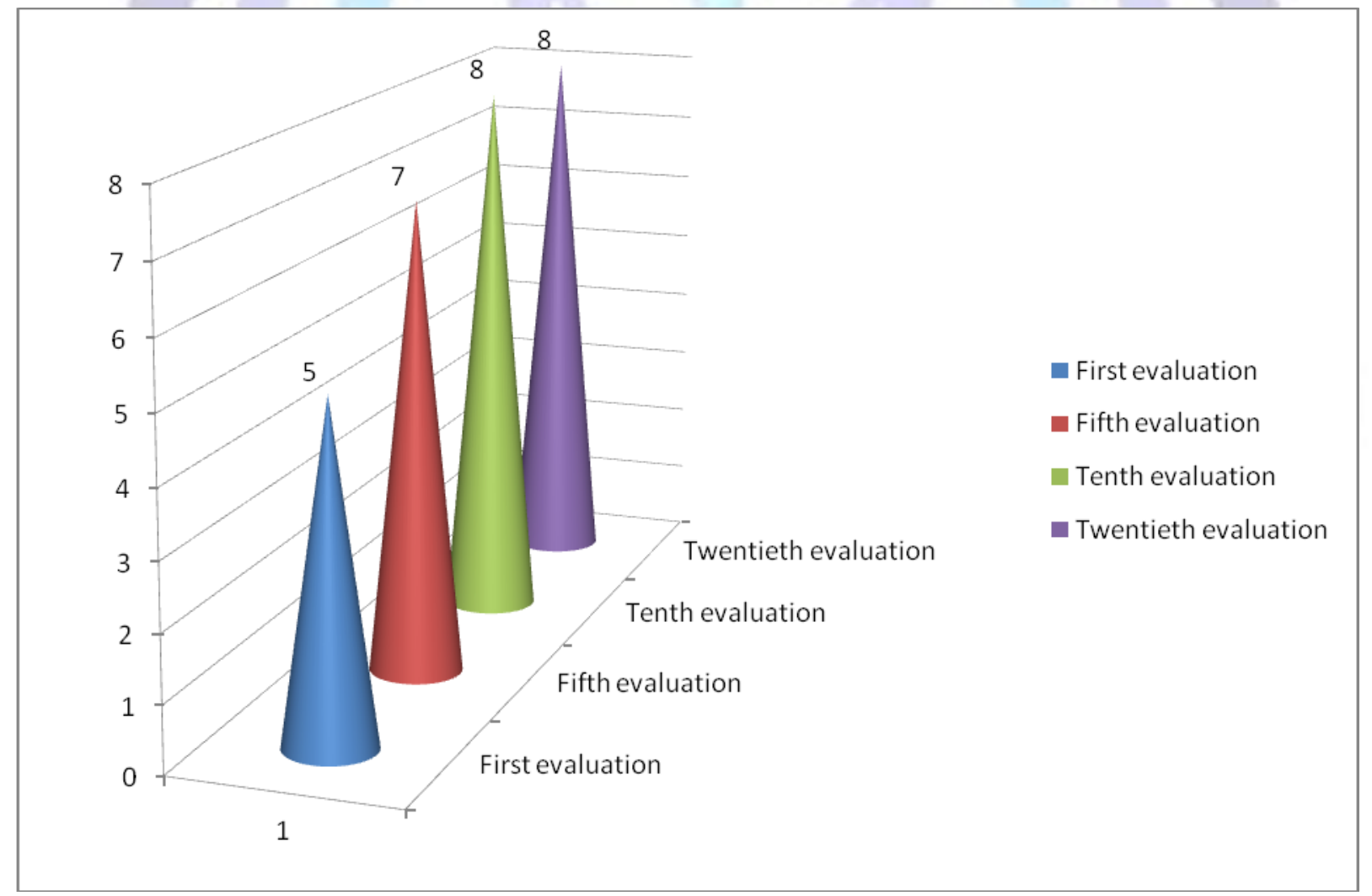

Graph n.2 


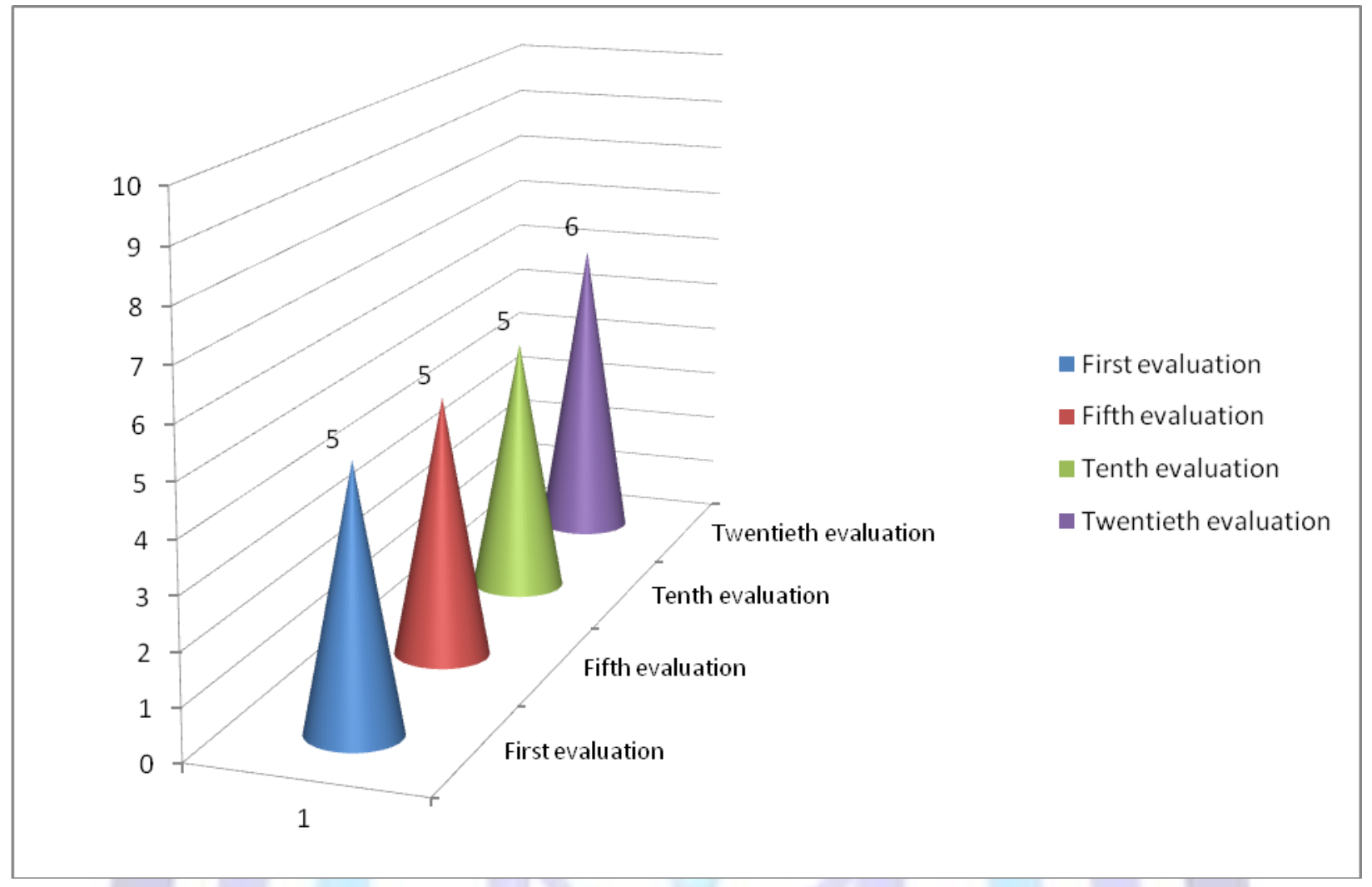

\section{Table 3 -Evaluation with video analysis and motor imagery}

Experimental group: evaluation with video analysis and motor imagery Name of difficulty Value of difficulty Score from 0 to 10 Deduction $1 / 2$ tuck jump $1 / 2$ 0,4 9

Control group: evaluation without video analysis and motor imagery Name of difficulty Value of difficulty Score from 0 to 10 Deduction $1 / 2$ tuck jump $1 / 2$ 0,4 8 TOT. 0

\section{Discussion and Conclusions}

The results highlight the important aspects that very often in sports, and in particular in Aerobics, are ignored: the introduction of video analysis and ecological approach by motor imagery during workouts. Often people think that all this can only be a waste of time that takes away space for the preparation of athletes. These findings are an example of how one might proceed in this direction having the same results, sometimes even better, than those who train continuously without video support.

The athlete of the experimental group improved by 0.20 compared to the first evaluation, while the athlete evaluated without video analysis has improved only by 0.10 and less rapidly than the experimental athlete.

Also a good practice of motor imagery has allowed the experimental athlete further improve by increasing the score of another 0.10. Experimental studies have demonstrated the validity of motor imagery in motor learning. A good practice can lead to the same results of hours of training. It is interesting to be able to continue the study of other athletes in the senior category to see whether the video analysis and the motor imagery are effective also for the evaluation of elements with greater values than those analyzed so far. Given the complexity of the elements of aerobics and the speed at which they run, the introduction of video analysis and motor imagery is essential in order to bring clear improvements in the method of evaluation of technical training, of the judges in the contest and athletes who reach greater awareness of their errors and greater mastery of their movements through motor imagery. Traditionally, the trainer is the unique subject to 
evaluate and assess the performance. New technology together new scientific paradigm on motor control and learning can open a new perspective on sport science. Particularly, the athletes should be always involved in training process and monitoring and evaluating will be a good practices of everybody take part in training.

\section{Refereces}

[1] Code of scores, http://www.federginnastica.it (2012) Roma , Italy

[2] Hughes, M., Bartlett, R. , 2002, Special edition on performance analysis. Journal of Sports Sciences, 20, 735-737. UK

[3] Hughes, M.D., Franks, I.M. (2004), National analysis of Sport 2nd Edition- better system for improving coaching and performance, London: E. \& F.N. Spon. UK

[4] Raiola G, Giugno Y, Napolitano S (2014). Self-evaluation by athletes on video analysis and motor imagery in aerobics. Procedia: Social \& Behavioral Sciences, vol. 117, p. 431-435, ISSN: 1877-0428 